\title{
Automatic gauge control hydraulic cylinder state identification using modified image based acoustic emission profile
}

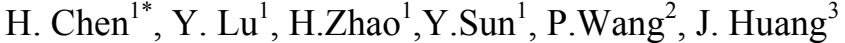 \\ ${ }^{1}$ State Key Laboratory of Hybrid Process Industry Automation System and Equipment Technology, Automation Research \\ and Design Institute of Metallurgical Industry, Beijing, China. P. R, 100071. \\ ${ }^{2}$ Power Electronic and Motor Drive Engineering Research Center, North China University of Technology, Beijing, China. P. \\ R, 100144. \\ ${ }^{3}$ Key Laboratory of Complex System Intelligent Control and Decision (Ministry of Education), School of Automation, \\ Beijing Institute of Technology, Beijing, China. P. R, 100081. \\ "Corresponding Author, Phone: 86-10-83802363, Fax: 86-10-63851580, Email: Shadow_c3186@msn.com
}

\begin{abstract}
The growing requirements of steel products qualities bring even tricky restrictions to the rolling process. With the fast response, reliable control and low maintenance requirements, the hydraulic automatic gauge control system has been widely applied to screw down the roller to maintain the precise rolling spaces for product quality control. Regarding to non-stopped heavy duties charged to the system, unavoidable faults and disfunctions not only influence the product quality, but also bring underlying safety issues. The hydraulic cylinder is the executing component of the roller screw down and it has the dominant percentage of hydraulic components' faults. Working with unexpect loading is one of major impact factor that causes several chain-effects happened to the cylinder, as the classical diagnostic process is lack of cross-validating and time-consuming, the paper proposes the potentials of using acoustic emission to fill the dilemmas. The works include the data acquisition process to record the ultrasound acoustic signals from the hydraulic cylinder during it was loaded with 6 types of conditions, a modified image based acoustic emission approach constructed by using 8 significant waveform features was applied to generate visual effects and transform the cylinder acoustic emission signals under various loadings to a uniform format, the subtle differences among various loadings can be observed based on the pixel and intensity changes of the images. By applying the principal component analysis to project the acoustic emission image profiles onto the $3 \mathrm{D}$ plane, a clear trajectory can be observed with normal and overload conditions allocated upon the positive and negative sides of the axis. The result provided not only the potential of using acoustic emission for dynamic state identification of the subtle changes, but also opens up the possibility of preventive measures to the cylinder at risks in the future.
\end{abstract}

Keywords-acoustic emission; hydraulic cylinder; automatic gauge control; image based profile; state identification;

\section{INTRODUCTION}

The growing industry requires extreme quality of hot and cold rolling steel products such as slabs, metals and foils, which brings even tricky accuracy-requirements to the rolling process to maintain the manufacturing tolerances under the acceptable condition [1]. According to this situation, the precision of the space between the rolls is considered to be one of key issue to minimize the product manufacturing tolerances. With the fast response, high level of control accuracy, reliable pressure overload protection, low maintenance, and ability to perform the continuous position control under the heavy loads and complicate force conditions, the hydraulic automatic gauge control (HAGC) systems have been widely applied in the steel rolling processes [2].

Although most of the HAGC systems are currently working with little maintenances thanks to the well-design of the HAGC system and less displacements of the hydraulic cylinder which is the executing component of the HAGC system, with the non-stop working of the rolling mills, the disfunctions and faults are unavoidable. Previous studies showed a set of researches and contributions regarding to HAGC state identification and fault diagnostics [3-7], the works include the object-oriented approach for obtaining fault knowledge on HAGC system followed by the development of adaptive fault diagnostic technique based on enlarged neural network to deal with the pressure change forecasting of the strip mill HAGC system [3-4]; the design of a decoupling subsystem based on differential geometric approach for robust diagnostic of load uncertainties [5]; as well as the innovative design of optimized BP neural work for dealing with one of major HAGC system failure named multi-sensor faults [6-7].

According to the official statistics revealed from one of Chinese major steel-producer, the hydraulic cylinder dominants over $33 \%$ of hydraulic components faults [8], however, the above mentioned researches were mainly focused on the fault detection and diagnosis of the HAGC control system, only a little works investigated the hydraulic components such as hydraulic cylinder [9-10], as the key component that makes direct contact with the working rolls to justify the rolling space, the abnormal condition of cylinder could directly influence the rolling process and bring underlying safety issues, a nonlinear model based adaptive robust observer was designed for fault detection and diagnosis of typical faults of electro-hydraulic cylinder (EHC) with verification supported by the simulation results [9]. Unexpected loading of the cylinder is considered as a major impact factor of disfunction of HAGC hydraulic screw down [8], which could caused cylinder faults such as leakages or seals broken, the current state identification process of insufficient loading is based on the reading and processing of the pressure signals, as the direct reading from the pressure 
monitor only able to provide the static display of the working pressure and the pressure signals are sensitive to fluctuations and noises [10], to some extent it provides helpless suggestions to the engineers in terms of disfunction spotting, a wavelet energy [11] based approach was derived to extract significant features from the pressure signals acquired from the field and acted as the inputs to BP neural network [12] with the unexpected loading caused by the inner leakage being correctly identified [10], however, with wavelet energy and neural network approach employed to deal with the noise issue, large computational burden could be charged to the diagnostic system.

As a mature non-destructive testing and evaluation technique, acoustic emission (AE) has been implemented in several areas for state identification and health monitoring [13] regarding to its subtle changes sensitivity and noise robustness in the microscopic level [14]. With an exploratory study of identifying the pressure differences in the hydraulic cylinder until failure can be considered as to determine subtle tolerances of the cylinder during its still under health condition, AE could be a useful option to meet the research requirement thanks to its main advantages.

The paper presents a modified AE based approach for state identification of the hydraulic cylinder, thereby demonstrating the potential of using $\mathrm{AE}$ for quick health monitoring of the cylinder as well as providing the crossvalidation option for dynamic fault detection. The experimental setup is introduced in section II. Section III devotes to the AE signal processing methodology based on the modified image based profile that the original model was previously introduced by the authors in the biomedical engineering researches. By creating the image based profiles of the AE signal emitted from the cylinder under various loadings, and further analyzing by the principal component analysis (PCA), the performance of the AE signal processing approach is discussed in section IV. Concluding remarks and discussion of the potential future works is given in section VI.

\section{EXPERIMENTAL SETUP}

The object under examination is a HAGC hydraulic cylinder with dimensionality $\mathrm{R}=550 \mathrm{~mm} \times \mathrm{I}=600 \mathrm{~mm} \times$ $\mathrm{O}=850 \mathrm{~mm}(\mathrm{R}, \mathrm{I}, \mathrm{O}$ are referred to as diameter of piston rod, as well as the inner and outer diameter of the cylinder, respectively) and with maximum $25 \mathrm{MPa}$ working load designed. It is used for $660 \mathrm{~mm}$ hot rolling mill screw down, the cylinder is made by the carbon steel except the piston seal which is made by the polytetrafluoroethylene.

The data acquisition system set-up is shown in fig. 1 . The AE signals emitted from the cylinder were captured and amplified by five piezoelectric transducers (PAC-R15i, frequency response $100-400 \mathrm{KHz}, 40 \mathrm{~dB}$ gain pre-amplifier integrated) were fixed on the cylinder by using the magnetic clips, the reason of using a wide-band sensor is to provide a better record of the new signals from an unknown process (in this case the exact source and actual mechanisms of $\mathrm{AE}$ are unknown yet) [13], with 4 sensors were mounted on the surface of cylinder where the position is the same as the cylinder piston refer to the ground (around $25 \mathrm{~mm}$ higher apart from the ground), each adjacent pair of sensors having around $90^{\circ}$ with reference to the cylinder center if considering the cylinder surface where the sensors attached as a circle. Another one sensor that acts as a filtering sensor was attached on the place just nearby the fuel inlet. Signals acquired were then recorded and pre-processed by using the SH-II AE condition monitoring system supported by the Physical Acoustic Corporation (PAC), and finally stored in a Lenovo-Thinkpad-T420 laptop computer.

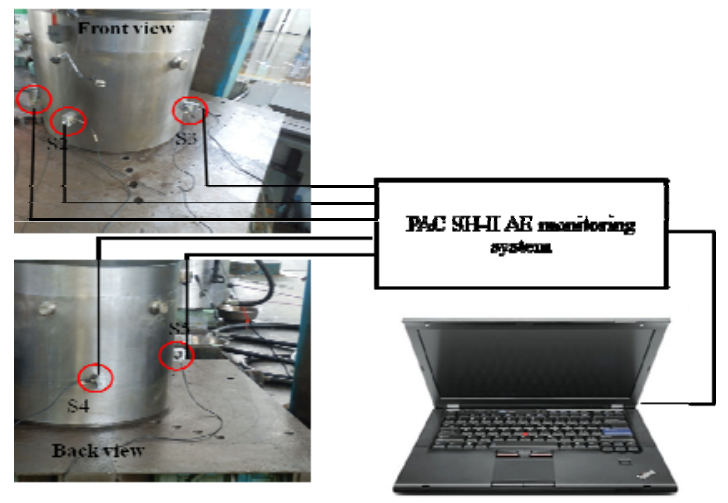

Fig. 1 Data acquisition system

Each individual $\mathrm{AE}$ hit was recorded with $1 \mathrm{MHz}$ sampling frequency within $2 \mathrm{~ms}$ time interval, a $40 \mathrm{~dB}$ detection threshold was employed according to the pencil lead break testing result, the peak definition time (PDT), hit definition time (HDT) and hit lockout time (HLT) are fixed to $200 \mu \mathrm{s}, 800 \mu \mathrm{s}$ and $1000 \mu \mathrm{s}$, respectively to enable better signal recording. As it is an exploratory study of $\mathrm{AE}$ emitted from the HAGC hydraulic cylinder, the data acquisition protocol are determined according to the ASTM E650/650M-12 and ASTM E1930/1930M-12 standards [1516]. Prior to record $\mathrm{AE}$, the cylinder is placed on the standard $45 \mathrm{MPa}$ hydraulic testing bed which consists of a rolling mill housing on top of the testing kit and a bearing cylinder on the bottom as well as the corresponding supplies to simulate the actual working environment of a rolling mill hydraulic screw down. The AE events were created by pumping the hydraulic oils into the cylinder to be tested to make the object reaching $5 \mathrm{MPa}, 10 \mathrm{MPa}, 15 \mathrm{MPa}, 20 \mathrm{MPa}$, $25 \mathrm{MPa}$, and $35 \mathrm{Mpa}$, respectively, and holding each condition for 100 seconds individually. Prior to testing, the actual pressure were monitored by the pressure scale and the actual volumes of hydraulic oils pumped into the cylinder from the hydraulic station, thereby ensuring the cylinder is under precise loading.

\section{INTRODUCTION OF IMAGE BASED ACOUSTIC EMISSION PROFILE}

As there are vast majorities (in this study, maximum number of $\mathrm{AE}$ events $>14000$ hits were acquired when the cylinder was loaded at $20 \mathrm{MPa}$ ) of $\mathrm{AE}$ bursts created by the cylinder under various loading conditions, direct separation of different loading conditions using the whole waveforms 
or feature set is difficult. In order to reduce the dimensionalities of the AE signals, convert the AE signals emitted from each loading condition to a uniform format, as well as to create the visual effect for further inspection of the cylinder under various loadings, a modified image based AE profile, namely the hits density approach is proposed. The hits density approach and its applications were previously introduced by the authors since 2009 [17-21], it is a type of multivariate statistical based methodology that re-classifying the number of $\mathrm{AE}$ events by using the specified $\mathrm{AE}$ feature combinations within various value granularities. The mathematical representation of this approach is given by (1)

$$
\mathbf{N}(F, \psi)=F_{\psi_{j}}\left(V_{1}, V_{2}, \ldots V_{i}\right)
$$

where, $\mathbf{N}$ denotes the number of AE events constrained by the specific AE feature combination, $V_{1}, V_{2}, \cdots, V_{i}$ are referred to as the selected features; $F_{\psi_{j}}$ denotes the number of $\mathrm{AE}$ events which satisfies one of particular $\mathrm{AE}$ feature combinations [17].

With the quick examination purpose of the cylinder states by using the AE technique in a pilot study, the visual effect of $\mathrm{AE}$ hits density based on 2D image is taken into account. The image that represents the cylinder under a particular working status can be segmented into four quadrants by using two axis radiated via the horizontal and vertical directions from the center of image with each quadrant represents one of particular $\mathrm{AE}$ feature combination. As illustrated in fig. 2, the $2 \mathrm{D}$ image based $\mathrm{AE}$ representation allows a maximum of $4 \mathrm{AE}$ feature combinations to be displayed in four quadrants determined by $\psi_{j}$ and each pixel within $\psi_{j}$ represents the number of AE events retained by a particular feature class determined by not only the feature types, but also the value intervals. The directions of feature classes with increasing values are oriented outwards from the image center to enable visualization of pattern symmetry. The AE hits density in each quadrant can be represented by using (2)

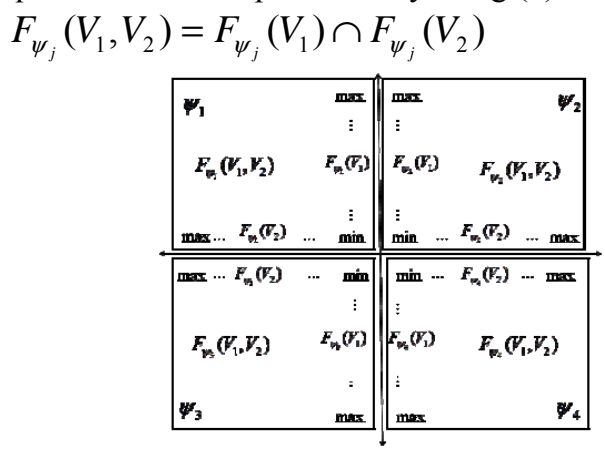

Fig. 2 Image based representation of AE signals where, $F_{\psi_{j}}\left(V_{1}\right)$ and $F_{\psi_{j}}\left(V_{2}\right)$ are referred to as the selected feature vector in quadrant $\psi_{j}$, respectively.

\section{EXPERIMENTAL RESULT}

This section discusses the signal processing result of AE emitted from the HAGC hydraulic cylinder using the methodology discussed in section III. Prior to create the image based profiles, the $\mathrm{AE}$ feature vector consists of traditional AE descriptors [13] and statistical moments [22] of each individual AE waveform were calculated. Through the statistical distribution analysis which aids the feature selection for AE hits density creating, 8 waveform features showing statistical significancy are selected, namely, amplitude, average signal level above the detection threshold (ASLOT), counts, duration, energy, average signal level over the whole AE wave (ASLOW), rise-time, and counts-to-peak (CTP), respectively. The suggestions of feature selection can be found in authors' previous papers [20-21], in order to save the spaces in this paper, feature selection advices are omitted.

\section{A. Intervals setting of the selected features}

Fig. 3 gives an example of $\mathrm{AE}$ waveform with a portion of $\mathrm{AE}$ features labeled, the amplitude is the peak amplitude (positive or negative) of each $\mathrm{AE}$ waveform; duration and rise-time are referred to as the time interval of the $\mathrm{AE}$ pulses exceeding the detection threshold and the time interval between the $1^{\text {st }}$ threshold crossing and the $\mathrm{AE}$ peak, respectively; counts and CTP are referred to as the number of $\mathrm{AE}$ pulses exceeding the detection threshold, and the $\mathrm{AE}$ counts between the $1^{\text {st }}$ threshold crossing and the $\mathrm{AE}$ peak, respectively; Unlike the traditional $\mathrm{AE}$ feature definitions, which using the average signal level (ASL) to assess the continuous varying of AE events generated by the subject under testing, the calculation of two ASL terms (i.e. ASLOT and ASLOW) are focused on the instantaneous varying, with ASLOT represents the average amplitude over the duration and ASLOW represents the average amplitude over the whole waveform while an $\mathrm{AE}$ event is being identified.

By dividing the selected AE features into four pairs, the AE signals generated by the cylinder under each loading condition mentioned in section II can be transformed to a 2D image, with the left top quadrant of the image represents number of AE events identified using amplitude and ASLOT, the feature combinations are changed to counts and duration, energy and ASLOW, as well as rise-time and CTP, respectively when the image sub-field moving clockwise from the $1^{\text {st }}$ quadrant to the $4^{\text {th }}$ quadrant, respectively. The feature granularities can be calculated using (3)

$$
\left\{\begin{array}{lc}
k(n) \leq F(V)<k(n+1) & n \in[1,6] \\
F(V)>k(n) & n>6
\end{array}\right.
$$


where, $k(n)$ is a vector consists of the value intervals of selected features. In quadrant $\psi_{1}$, the feature interval of amplitude is set to $k_{a m p}(n)=[40,50,60,70,75,80,85]^{\mathrm{T}}$, as the amplitude values showing more separation among various loading at the tail of distribution, the value interval between the $4^{\text {th }}$ and $7^{\text {th }}$ terms are set to $5 \mathrm{~dB}$, the feature interval of ASLOT is set to $k_{\text {ASLOT }}(n)=[0,10,20,30,40,50$, $60]^{\mathrm{T}}$. In quadrant $\psi_{2}$, the feature interval for AE counts and duration are set to $k_{\text {counts }}(n)=[1,250,500,750,900,1250$,

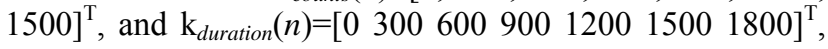
respectively. In quadrant $\psi_{3}$, the feature interval for energy is fixed to $k_{\text {energy }}=\left[0,10^{5}, 2 \times 10^{5}, 3 \times 10^{5}, 4 \times 10^{5}, 5 \times 10^{5}, 6\right.$ $\left.\times 10^{5}\right]^{\mathrm{T}}$ and $k_{\text {ASLOW }}=[0,10,20,30,35,40,45]^{\mathrm{T}}$ with the intervals within the last 3 terms set to $5 \mathrm{~dB}$ according to the statistical significant shown in the univariate statistical distribution [20]. In quadrant $\psi_{4}$, the feature interval for rise-time and CTP are set to $k_{\text {rise-time }}=[0,300600,900,1200$, $1500,1800]^{\mathrm{T}}$, and $k_{C T P}=\left[\begin{array}{lllllll}1 & 250 & 500 & 750 & 1000 & 1250 & 1500\end{array}\right]^{\mathrm{T}}$, respectively. Also with the symmetric visualization purpose, the intervals are set to the same value in each quadrant in this study, the robustness of feature granularities were discussed by [17] in the proceeding publication, which proofs the value interval affects little in the hits density model.

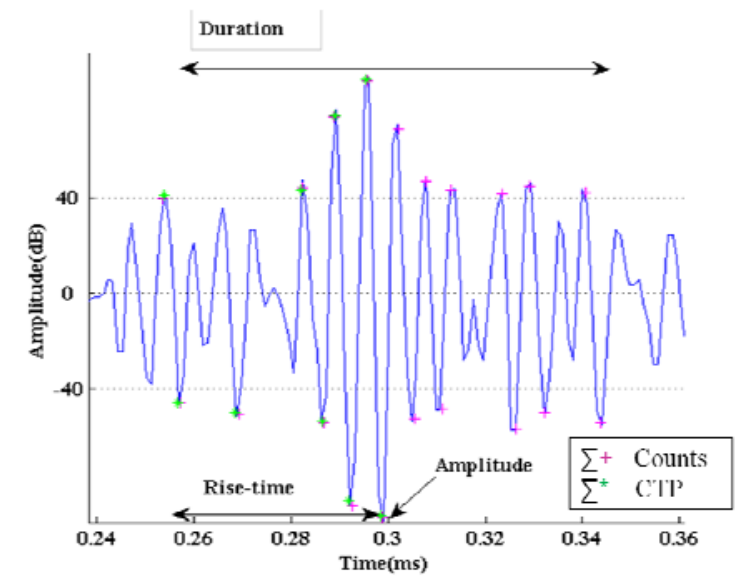

Fig. 3 An example of AE waveform emitted from the HAGC hydraulic cylinder with partial features labeled

\section{B. Construction and evaluation of the cylinder AE profiles}

By substituting the value intervals of the selected features to (2) and (3), the hits density of the cylinder under $5 \mathrm{MPa}, 10 \mathrm{MPa}, 15 \mathrm{MPa}, 20 \mathrm{MPa}, 25 \mathrm{MPa}$, and $35 \mathrm{Mpa}$ are shown in fig. 4. With the paper reports the possibility of using $\mathrm{AE}$ for hydraulic cylinder state identification, the comparative study of the signal emitted from various sensors will be discussed in the future publications, AE signals from sensor $\mathrm{S} 3$ with the maximum amount of $\mathrm{AE}$ events acquired are picked up for demonstration, as the hits density created by using the signals collected by another 3 sensors showed similar results. In order to simplify the analysis, AE signals generated by the transition of two adjacent pressure statuses (e.g. transition of $5 \mathrm{MPa}-10 \mathrm{MPa}$ ) are removed.

With the image based AE profiles demonstrate in fig. 4, it is seen that the cylinder provides the smallest amount of $\mathrm{AE}$ events among 6 loading conditions with the lowest
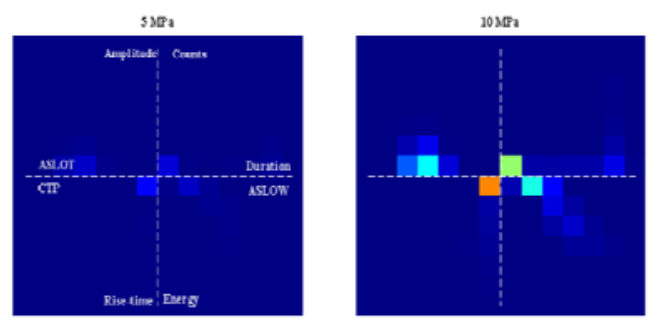

$15 \mathrm{MP}$
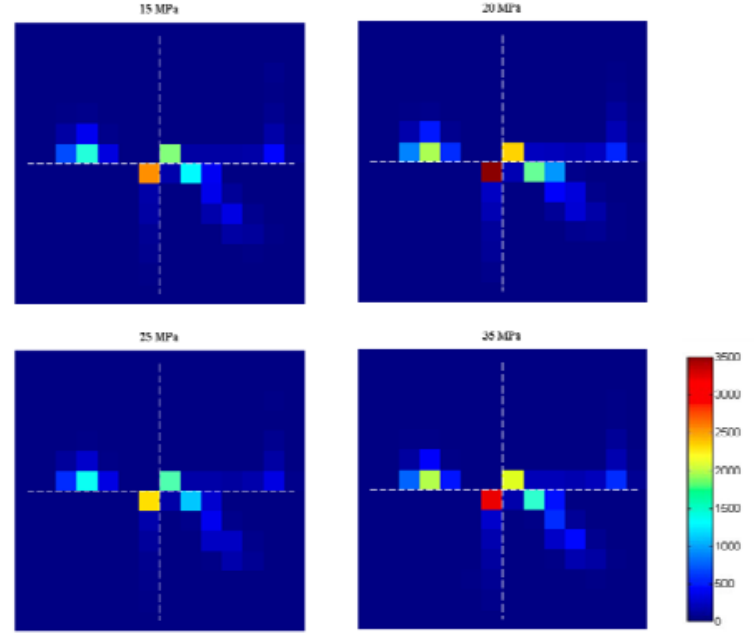

Fig. 4 Image based profiles for the cylinder under various pressures

signal levels that can be observed over four quadrants of the image when the cylinder pressure is pumped to $5 \mathrm{MPa}$. Types and amount of AE events are increased since the cylinder has been loaded to $10 \mathrm{MPa}$, and the main types of waveform are seen to remain unchanged while the pressure is increasing. However, the subtle differences can still be observed through the visual inspection of image based profiles. With the number of $\mathrm{AE}$ events constrained by $\mathrm{AE}$ counts and duration, it can be seen that although all pressure statuses provide similar type of AE signals (signals with counts lower than 250 and durations varied from 0 to 1800 $\mu \mathrm{s}$, and signals with duration longer than $1500 \mu \mathrm{s}$ and with counts varying from the minimum to maximum). The number of $\mathrm{AE}$ events with minimum duration and counts are increased from $10 \mathrm{MPa}$ to $20 \mathrm{MPa}$ and decreased from $25 \mathrm{MPa}$ to $35 \mathrm{Mpa}$, with the maximum amount of AE events seen to occur during the cylinder loading at $20 \mathrm{MPa}$, and with the number of AE hits reaches to around 2500. Another 
distinctive difference can be observed in the 4th quadrant of the image, for the AE signals with minimum CTP and the shortest rise-time, the number of $\mathrm{AE}$ events are seen to increase slightly from $10 \mathrm{MPa}$ to $20 \mathrm{MPa}$, and decreased when the cylinder is pumped to $25 \mathrm{MPa}$, and re-increased when the cylinder is loading at $35 \mathrm{MPa}$, with the maximum number of AE events seen in 20MPa. This scenario can be linked to the actual specification of the cylinder under examination, as the maximum working load of the cylinder is designed to $25 \mathrm{MPa}$, the aforementioned scenario could be understood as some underlying mechanical disfunctions of the cylinder that causes by the overloading, which may further brings failure to the cylinder during rolling process.

\section{Cylinder states identification by principal component analysis}

In order to further assess the sensitivity of $\mathrm{AE}$ as a potential signature for state identification of the HAGC hydraulic cylinder, the further multivariate analysis is needed. According to the previous studies produced by the authors, the principal component analysis (PCA) could be one of good option for further highlighting the dissimilarities between the image based profiles [17-21]. PCA is a kind of multivariate statistical based technique that has been widely used for dimensional reduction and feature extraction, which implements by projecting the multidimensional vector onto a new plane formed by the principal components (PC) through the singular value decomposition (SVD) and the matrix inner products with the projection of original matrix onto the $\mathrm{PC}$ space represents by the $\mathrm{PC}$ scores, thereby re-organizing the variables with higher variances onto the lower dimensional space [22].

Fig. 5 shows the PCA projection of the hits density based image profiles of AE events emitted from the HAGC cylinder under 6 different conditions with $98.86 \%$ of variances remained. The projection shows a clear trajectory of the cylinder pressure changes from $5 \mathrm{MPa}$ to $35 \mathrm{Mpa}$, it starts from $5 \mathrm{MPa}$ at the left extreme of the PCA space, pattern is moving towards the positive direction with the longest displacement when the cylinder pressure is increasing to $10 \mathrm{MPa}$, and it is moving through the positive direction over the $1^{\text {st }} \mathrm{PC}$ plane and negative direction over the $2^{\text {nd }}$ and $3^{\text {rd }}$ PC planes when the cylinder status is increasing to $15 \mathrm{MPa}$. Pattern is keep descending through the $2^{\text {nd }}$ and $3^{\text {rd }} \mathrm{PC}$ domain while the loads are increasing from $15 \mathrm{MPa}$ to $25 \mathrm{MPa}$ with the $1^{\text {st }} \mathrm{PC}$ scores remained nearly unchanged. The pattern is increased in the $1^{\text {st }} \mathrm{PC}$ domain when the cylinder status is approaching $35 \mathrm{MPa}$. As the projected trajectory shown in the figure, one of scenario is good to mention, with the cylinder pressure changes, the $1^{\text {st }}$ PC score only crossover the positive side once while the pressure is increased to $35 \mathrm{MPa}$, with the projection in the space formed by the $1^{\text {st }}$ PC weighted around $97 \%$ of total variance, this should show some statistical significancy. It could be related to the properties of PCA as well as the previous findings provided by the authors, where the projection of hits density patterns with significant differences would be distributed at two sides of origin [17, 20-21]. This also can be linked to the actual mechanisms of the cylinder, as the maximum $25 \mathrm{MPa}$ working load of cylinder is designed, only the overloaded condition distributed on the positive side means it may causes abnormal happens.

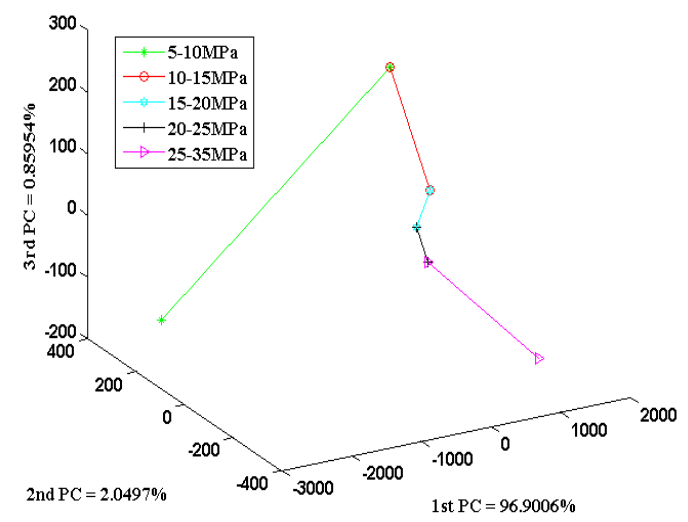

Fig. 5 3D PCA projection of cylinder AE profiles

The result showed in fig. 5 demonstrates the potential of using the hits density based AE combined with the PCA for dynamic state identification and assessment of HAGC hydraulic cylinder. By increasing the majority of AE signals related to more cylinder conditions, such as vibrating, leakage, etc, it will have possibility to establish a reference trajectory of the cylinder statuses developing from normal working condition to various abnormal or fault conditions through the further modification of image based AE profile, a $3 \mathrm{D}$ cube will be applied to bearing more $\mathrm{AE}$ feature combinations for describing the detail conditions as well as even subtle differences. The actual working condition of a cylinder and the possible developments could be examined based on the position of its projection on the PC domain along the trajectory and the distance of it with respect to each reference condition. Use of AE to assess the hydraulic cylinder statuses opens up a possibility to introduce not only the potential tool for dynamic monitoring of subtle state variation in the executing component of HAGC system, but also the preventive measures to the cylinder at risks.

\section{CONCLUSION AND DISCUSSION}

The works presented the potential of using $\mathrm{AE}$ as a signature to identify the dynamic statuses of the HAGC cylinder. By continuously loading 6 pressure statuses on the cylinder to generate $\mathrm{AE}$ and applying the hits density based AE signal processing approach consists of 8 significant features to the signals acquired, the image based cylinder AE profiles could be created for state identification, with the 
cylinder under $5 \mathrm{MPa}$ loading generated minimum level of $\mathrm{AE}$ events in terms of both amount and waveform types; signal classes were increased while the pressure was increased from $5 \mathrm{MPa}$ to $10 \mathrm{MPa}$, the waveform types which determined by selected feature combinations remain unchanged since the cylinder is working with the pressure higher than $10 \mathrm{MPa}$, whereas the majority of $\mathrm{AE}$ events in two particular waveform classes determined by counts and duration, as well as CTP and rise-time were seen to increase corresponding to the increasing pressures from $10 \mathrm{MPa}$ to $20 \mathrm{MPa}$ and decrease from $20 \mathrm{MPa}$ to $35 \mathrm{MPa}$, which could be related to the underlying mechanisms of the cylinder under examination. By projecting the $\mathrm{AE}$ profiles of the cylinder under various loadings onto the PCA space, a clear trajectory related to various pressures could be observed with trajectory moving from the negative axis towards the positive axis with overloading condition distributed in the positive side of the space formed by the $1^{\text {st }} \mathrm{PC}$.

The above mentioned results showed the pioneering work of using $\mathrm{AE}$ for state identification of HAGC hydraulic cylinder. In the future studies, additional tests will be applied to more types and conditions of cylinders with more abnormal conditions included, in order to record more AE signals for further studies; sensor attachment optimization methodology for the HAGC hydraulic cylinder will be investigated, thereby discovering the most suited sensor attachment solution for the cylinder; unsupervised feature selection criterion will be established based on the application of intelligent searching algorithms on the $\mathrm{AE}$ features derived, thereby selecting the most appropriate feature combinations to build the hits density profiles relating to various conditions of the cylinder; clustering analysis will be applied to dig out the dominant and distinctive $\mathrm{AE}$ waveforms for further supporting of the cylinder states identified by AE.

Regarding to the potential future works mentioned, the following issues are worth to discuss: in terms of the sensor attachment, with the current method based on the ASTM standards, which are referring to the standard sensor mounted solution and the AE applied in the storage tank, as the hydraulic cylinder can be treated as pressure storage, and the heavy duties equipments as well, the mathematical model of the deformation of the cylinder is necessary to be considered; With the cylinder works by pumping the oils to create pressures against the rolling mill housing, and in this study, the sensors were not attached spatially, the exact source of $\mathrm{AE}$ in the cylinder is difficult to identify, regarding to the actual mechanisms of AE signal generation, the AE signals could be emitted during the pressures loaded on the inner surface of cylinder, or by the piston squeezing the piston seal, it could also be generated by the laxity or other subtle changes on the joints or the weldings, although the often used time-of-arriving (TOA) approach [23] able to localize the source of $\mathrm{AE}$, it is not specified for the cylinder mechanism purposes, the integration of additional visual based measurement modality may be the alternative to deal with this issue; in terms of the cylinder condition monitoring, as well as the AE waveform classification, it is more relying on the pattern recognition techniques, however, with neither the classical knowledge based recognition methodology, nor the modern data-driven based approach is seen to provide the perfect classification performance, the combination of both sides could be a better option for dealing with the health monitoring problem of the even complex mechanical design.

\section{ACKNOWLEDGMENT}

The work is supported by state key development program for basic research of China (Grant ref: 2012CB724304), and we would also like to extend our gratitude to Prof. L-K Shark from University of Central Lancashire, UK, Dr. Benoit Mascaro from France, and Physical Acoustics Corporation Beijing office for their technique inputs.

\section{CONFLICT OF INTERESTS STATEMENT}

None.

\section{REFERENCES}

[1] A. Stepanov, S. Zinchenko, S. Efimov, V. Ordin, M. Filatov, et al. "Main trends in the growth of converter steelmaking at the company severstal", Metallurgist, Vol. 49(9/10), 2005, pp. 380-2, doi:10.1007/s11015-006-0010-0.

[2] H. Liu. "Application of predicted extrapolation control strategy in hot strip rolling mills gauge system", Proc. World Automat Cong (WAC), Jun, 2012, pp. 1-4.

[3] H. Wang, Y. Rong, J. Cui, and S. Liu, "Study on knowledge processing of fault diagnosis for hydraulic AGC system", $2^{\text {nd }}$ IEEE Intl Conf Infor Man Eng (ICIME), Apr, 2010, pp. 1-3, doi:10.1109/icime.2010.5477802.

[4] H. Wang, Y. Rong, S. Liu, and J. Cui, "Identification for hydraulic AGC system of strip mill based on neural networks", Intl Conf Comput Desgn Appl (ICCDA), Jun, 2010, pp. V2-377-80, doi:10.1109/iccda.2010.5541406.

[5] M. Dong, C. Liu, and G. Li, "Robust fault diagnosis based on nonlinear model of hydraulic gauge control system on rolling mill", IEEE Trans Contr Syst Tech, vol. 18(2), 2010, pp. 510-5, doi:10.1109/tcst.2009.2019750.

[6] X. Wang, C. Liu, and M. Li, "Sensors fault diagnosis of hydraulic automatic gauge control system based on wavelet neural network", IEEE Intl Conf Electr Contr Eng (ICECE), Jun, 2010, pp. 3009-12, doi: 10.1109/iCECE.2010.732.

[7] X. Wang and K. Zhang, "Sensors fault diagnosis of hydraulic automatic gauge control system based on neural network optimized by genetic algorithm", IEEE Intl Conf Oxide Mater Electr Eng (OMEE), Sept, 2012, pp. 114-7, doi:10.1109/omee.2012.6343516.

[8] W. M.Ye, Z. Xu, and K. Deng, "The fault and diagnosis for hydraulic system of metallurgical machinery", China High Tech Enterp, Dec, 2008, pp. 124-125. (Chinese)

[9] P. Garimella and B. Yao, "Model based fault detection of an electrohydraulic cylinder", Proc. Amer Contr Conf, Vol. 1, Jun, 2005, pp. 484-9, doi: 10.1109/acc.2005.1469982.

[10] L. Zhang, C. Zhang, and T. Shi, "Inner leakage fault diagnosis of hydraulic cylinder using wavelet energy", Adv Mater Res, vol. 139141, 2010, pp. 2517-21, doi: 10.4028/www.scientific.net/AMR.139141.2517 .

[11] S. Qian and D. Chen, "Joint time frequency analysis: methods and applications”, Prentice-Hall, 1996.

[12] C. M. Bishop, "Neural networks for pattern recognition”, Clarendon Presss, 1995. 
[13] H. Chen, "Discovery of acoustic emission based biomarker for quantitative assessment of knee joint ageing and degeneration", in computing, engineering and physical science. Ph.D Thesis, Preston: University of Central Lancashire, 2011.

[14] C. K. Tan, P. Irving, and D. Mba, "A comparative experimental study on the diagnostic and prognostic capabilities of acoustics emission, vibration and spectrometric oil analysis for spur gears", Mech Syst Signal Pr, vol. 21(1), 2007, pp. 208-33, doi: 10.1016/j.ymssp.2005.09.015.

[15] ASTM Standard E650/650M, 2012, "Standard guide for mounting piezoelectric acoustic emission sensors", ASTM International, West Conshohocken, PA, 2012, doi: 10.1520/E0650_E0650M-12, www.astm.org.

[16] ASTM Standard E1930 / E1930M, 2012, "Standard practice for examination of liquid-filled atmospheric and low-pressure metal storage tanks using acoustic emission", ASTM International, West Conshohocken, PA, 2012, doi: 10.1520/E1930_E1930M-12, www.astm.org.

[17] B. Mascaro, J. Prior, L. K. Shark, J. Selfe, P. Cole, et al. "Exploratory study of a non-invasive method based on acoustic emission for assessing the dynamic integrity of knee joints", Med Eng Phy, vol. 31(8), 2009, pp. 1013-22, doi: 10.1016/j.medengphy.2009.06.007.

[18] L. K. Shark, H. Chen, and J. Goodacre, "Discovering differences in acoustic emission between healthy and osteoarthritic knees using a four-phase model of sit-stand-sit movements", J Open Med Infor, vol. 4, 2010, pp. 116-25, doi:10.2174/1874431101004010116.

[19] L. K. Shark, H. Chen, and J. Goodacre, "Knee acoustic emission: A clue to joint ageing and failure", Rheumatology, 49, I79, 2010, doi: 10.1093/rheumatology/keq722.

[20] L. K. Shark, H. Chen, and J. Goodacre, "Knee acoustic emission: A potential biomarker for quantitative assessment of joint ageing and degeneration", Med Eng Phy, 33(5), 2011, 534-45, doi:10.1016/j.medengphy.2010.12.009.

[21] H.Chen, Y. Lu, L. Wang, "Analysis of dynamic acoustic emission signals using multivariate statistical technique for smaller dataset", J Vibr Meas Diag, 2013, in press (Chinese)

[22] I.T. Jolliffe, "Principal component analysis", Springer-Verlag, 1986.

[23] V. Salinas, Y. Vargas, J. Ruzzante, and L. Gaete, "Localization algorithm for acoustic emission", Phys Procedia, vol. 3(1), 2010, pp. 863-71,. 